\title{
EDITORIAL
}

\section{Highlight dementia risk to reduce CVD}

C onvincing patients to substantially change their lifestyle and, thus, reduce their risk of cardiovascular disease is often challenging. Although they understand the cardiovascular benefits of life-style change, they just can't bring themselves to give up some of the creature comforts they have become used to over the years-eating sugary and fatty food, smoking cigarettes, and watching television instead of going for a walk, run or bicycle ride, are a normal part of life for many. Additionally, getting patients to adhere to preventative medication regimens (for example, aspirin, a statin, and an angiotensin-converting-enzyme [ACE] inhibitor) is difficult even for those known to have coronary artery disease, including individuals who have had a previous myocardial infarction.

To most, degeneration in cognitive function is a far worse prospect than traditional cardiovascular diseasethe idea of not remembering who our loved ones are or what we did a few minutes ago is much scarier than the thought of having a 'heart attack' or developing another type of cardiovascular disease, which can probably be treated with the numerous therapies and interventions available today. Risk of dementia and cardiovascular disease are well known to increase with age and, given that the global population is aging, the incidence of these diseases are expected to rise over the next few decades.

Interestingly, evidence for a link between cardiovascular risk factors and dementia has been accumulating over the past few years; hypertension is now a well-recognized risk factor for degeneration of cognitive function and evidence for links between dementia and other cardiovascular risk factors, such as hypercholesterolemia and diabetes, is also available. Informing patients of these associations might aid in convincing them to adhere to prescribed lifestyle changes and medication regimens aimed at reducing these risk factors. We can now appeal to patients' heads and hearts when discussing the importance of reducing their cardiovascular risk factors.

Numerous longitudinal and cross-sectional studies have shown an association between uncontrolled hypertension and reduced cognitive function. Multiple studies have also shown that most antihypertensive therapies positively impact on the cognitive function of patients. In a longitudinal analysis of data from medical records of patients who had taken various antihypertensive medications, diuretics, $\beta$-blockers and ACE inhibitors were associated with reduced decline in cognitive function, and angiotensin receptor blockers (ARBs) were linked to improved cognitive function (Hajjar, I. et al. J. Gerontol. A. Biol. Sci. Med. Sci. 60, 67-73; 2005). More recently, a large, prospective cohort analysis has indicated that ACE-inhibitor and $\mathrm{ARB}$ use are associated with reduced incidence and progression of dementia, with ARBs providing the strongest effect (Li, N. N. et al. BMJ 340, b5464; 2010). Mechanisms of hypertension-induced degeneration of cognitive function are currently being elucidated, but cerebral hypoperfusion and chronic oxygen deprivation are thought to be involved. On a daily basis, clinicians find small punctated areas of brain hypoperfusion or microinfarctions in hypertensive individuals undergoing MRI.

Mutation in the gene for apolipoprotein E, a protein involved in cholesterol metabolism, has been linked to the development of Alzheimer's disease, and midlife hypercholesterolemia has been linked to increased risk of this disease in multiple studies in the past 10-15 years. Observational studies have shown an association between statin use and reduced risk of Alzheimer's disease, but prospective, randomized, controlled clinical trials have provided conflicting results. A proof-of-concept trial demonstrated that atorvastatin slowed cognitive decline in patients with mild-to-moderate Alzheimer's disease (Sparks, D. L. et al. Curr. Alzheimer Res. 2, 343-353; 2005); however, a later, larger, multicenter trial showed that there were no atorvastatin-induced effects on cognition in the same type of patients (Feldman, H. H. et al. Neurology 74, 956-964; 2010). Further randomized, controlled trials are, therefore, needed to determine whether or not statins confer this neurological benefit.

Diabetes is also thought to increase risk of Alzheimer's disease and potential mechanisms of diabetes-induced degeneration of cognitive function are currently being studied. Treatment with agonists of the peroxisome proliferator activated receptor, which stimulate insulinresponsive gene transcription, have prevented cognitive decline in some patients with the disorder (Risner, M. E. et al. Pharmacogenomics J. 6, 246-254; 2006).

We are all confronted with the difficult task of patient nonadherence to prescribed preventative medical regimens and lifestyle changes. Perhaps now is the time to capitalize on patients' concerns about future dementia as a means of improving their cardiovascular health. If we pass on our knowledge of dementia risk to patients when highlighting the importance of long-term lifestyle change and medication adherence, we might get more-lasting reductions in their bad habits.

doi:10.1038/nrcardio.2010.37
YWe can

now appeal

to patients'

heads and

hearts when

discussing the

importance

of reducing their [CV] risk factors 77

Bryony M. Mearns is the Editor and Valentin Fuster is the Editor-in-Chief of Nature Reviews Cardiology

Competing interests The authors declare no competing interests. 